Strategy for change The results were disseminated to the doctors and then again to the malaria quality care committee. Ninety nine percent $(99 \%)$ of clinical staff received training on the new guidelines; new laboratory forms were introduced to aid the rapid diagnosis of malaria at triage and in the emergency room and every ward had malaria job aid posters. The training of all staff took approximately 5 weeks to complete.

Measurement of improvement Using the same data collection methods, 40 clinical notes per week were selected, from a total of 5 weeks (Weeks 6-10); these weeks represented the start of the training/implementation; half way through training; end of training of all staff and 2 further follow on weeks after all training had been completed.

Over a period of 6 months: The proportion of patients with fever tested for malaria increased from $76 \%$ to $94 \%$. The proportion of malaria cases correctly classified according to the admitting doctor increased from 52\% to 94\%. The proportion of severe malaria cases given appropriate first line treatment increased from $50 \%$ to $95 \%$. Overall mortality attributed to Malaria decreased from $38 \%$ to $25 \%$

Effects of changes The improvement resulted in a higher quality of care being delivered to patients with malaria, in particular, better access to diagnostic tests, more accurate classification of malaria, improved documentation of malaria medication on prescription charts and improved sign off of doses given.

Lessons learnt The data collection process used (i.e. retrospective collection of a random selection on notes) may have given us biassed results and affected the quality of the outcome data. It was also difficult to measure certain specific areas of improvement. Prospective assessment and data collection would be a better way to measure the effects of change.

Message for others By engaging professionals, senior and junior, who are all involved in the care of a child and placing the child at the centre of the process and maintaining that focus on the child is an important way to improve the quality of their care, even in low resource settings.

\section{G523(P) THE PAEDIATRIC RETURN TO WORK PROGRAMME}

A Copeman, A Belfitt. Paediatrics, New Cross Hospital, Wolverhampton, UK

\subsection{6/archdischild-2015-308599.474}

Context We developed a half day programme of fully immersive simulation in a purpose built high fidelity centre to address confidence and training needs of paediatric ST doctors returning to clinical work after a break in practice.

Problem Returning to work after a break from clinical practice is daunting. Many trainees have up to a year away from clinical work on maternity leave or out of programme experience (OOP). Trainees undertaking research have up to 3 years away. Feeling under-confident, stressed and de-skilled can result in underperformance which ultimately puts patient safety at risk.

Assessment of problem and analysis of its causes Research suggests that the length of time away from clinical practice is a key factor affecting a doctor's performance when returning to practice - the longer the time out of clinical medicine in years the poorer their performance. $67 \%$ of doctors absent from practice for more than 18 months require moderate to considerable reeducation or training.

The RCPCH has written guidence to support trainees returning to work however simulation as a tool facilitating this process does not feature. We conducted a literature review but were unable to find any stand alone UK simulation programmes specifically aimed at helping paediatricians return to clinical practice safely.

Intervention A Paediatric Return To Work Simulation Programme was developed and piloted to address trainees confidence, skills and knowledge.

We used scenarios mapped to the RCPCH curriculum covering key areas of emergency management, safeguarding, leadership, communication skills and human factors.

Study design The effectiveness of our programme was assessed using pre and post programme questionnaires (Table 1). Two questionnaires were undertaken immediately before and after simulation training and a final questionnaire was undertaken 3 months later following their return to clinical practice.

Our primary outcome was aimed at evaluating the trainees confidence levels managing neonatal, paediatric and safeguarding problems and whether these improved following our simulation programme. We were also interested in the overall learning experience perceived by the trainees.

Strategy for change All OOP trainees within the deanery were emailed information about the pilot programme. 25 trainees participated in the pilot from September 2013-2014.

Measurement of improvement $88 \%$ of trainees expressed concern about returning to clinical practice prior to the simulation. Reasons given included lack of knowledge, technical skills, confidence, competence and senior responsibility.

Likert scales were used to assess confidence in managing acute paediatric, neonatal, and safeguarding problems pre and post intervention.

Results -confidence All trainees found the programme beneficial $100 \%$ found the training useful preparation for returning to work.

$100 \%$ agreed the experience learned from the training had been useful in their clinical work.

Free text responses were all overwhelmingly positive.

Effects of changes This programme improves confidence and the perceived ability of trainees to manage acute clinical problems. This positive effect appears to be sustained.

As a result of this pilot "The Paediatric Return to Work Simulation Programme" has secured funding and been approved regionally for trainees to attend prior to their return to clinical work.

Lessons learnt Organisations must have clear support and training in place to enable trainees to return to clinical work safely. Paediatric simulation can effectively facilitate this process. Future development would be aimed at identifying whether these positive effects result in an improvement in clinical practice and safety.

\begin{tabular}{|c|c|c|c|}
\hline & Pre Simulation & Post Simulation & 3 month follow up \\
\hline $\begin{array}{l}\text { Confidence managing } \\
\text { acute paediatrics }\end{array}$ & $28.00 \%$ & $96.00 \%$ & $100.00 \%$ \\
\hline $\begin{array}{l}\text { Confidence managing } \\
\text { neonates }\end{array}$ & $32.00 \%$ & $76.00 \%$ & $100.00 \%$ \\
\hline $\begin{array}{l}\text { Confidence managing } \\
\text { safeguarding }\end{array}$ & $44.00 \%$ & $92.00 \%$ & $75.00 \%$ \\
\hline
\end{tabular}


Message for others Simulation training programmes facilitating safe return to work should be available nationally and within other specialities.

\section{G524(P) JUNIOR DOCTOR ESSENTIALS: CRITICAL INCIDENT REPORTING}

${ }^{1} \mathrm{C}$ Parfitt, ${ }^{1,2} \mathrm{~L}$ Gabbott, ${ }^{3} \mathrm{~L}$ Hamilton, ${ }^{3} \mathrm{~K}$ Taylor. Paediatrics, Gloucestershire Royal Hospital, Gloucester, UK

10.1136/archdischild-2015-308599.475

Context The Junior Doctor Essentials (JDE) patient safety initiative was launched by a group of Junior Doctors, for Junior Doctors, in 2009. It has expanded across hospitals and specialties since.

Problem Despite national initiatives, the prevalence of inpatient iatrogenic harm remains high. Transition from student to foundation doctor is challenging; new job pressure, combined with clinical inexperience increases the risk of error. Transition from adult to paediatric medicine is equally difficult.

Empowering junior doctors to identify unsafe processes and implement mitigating solutions cultivates a patient safety culture. Junior Doctors Essentials, a junior doctor-led initiative, draws on their experiences to identify likely mistakes.

Assessment of problem and analysis of its causes At the project's inception, a focus group identified information foundation doctors considered would have eased transition into work. Their findings were augmented by organisational recommendations from The Medical Director. Since then, the model has been modified to place responsibility for designing the cards and gaining governance approval with locally nominated team leads, guided by the project team and informed by Critical Incident data. This has allowed the project to scale coherently across hospitals and departments nationwide.

Intervention Following review of departmental patient safety issues and Critical Incidents, ten double-sided 'credit-cards' highlight essential information. These are distributed to junior doctors at induction attached to a belt clip enabling portability and are immediately available for consultation in any situation.

Study design Junior Doctors are consulted annually to review each topic area's subjective effectiveness at protecting patient safety. More recently, the effect of the introduction of cards on Critical Incident rates was evaluated.

Strategy for change Survey results from the last four years have been presented to local, regional, national and international audiences. In presenting this formal critical incident data, we hope to expand the project further.

Measurement of improvement Local paediatric Critical Incident reports were evaluated pre- and post- JDE card introduction. We used strict inclusion and exclusion criteria to ensure we reviewed incidents that would involve the group of staff using the cards.

Critical Incident data from the year before the cards' introduction shows that $33 \%$ of incidents would have been covered by information on the cards. Over a one-year period since their introduction, only 1 incident (3.2\%) was covered.

It was incidentally noted that some Critical Incidents reported were a result of errors made by other teams with regular contact with our group of paediatric patients. We are considering expanding the cards locally.

We also detected recurrent incidents regarding prescribing errors and use of the DKA protocol. These will now likely be included in the next update of the cards.
Effects of changes Objectively, our review of Critical Incident data suggests the cards have made a genuine clinical impact.

Subjectively, the cards are well received by their target audience. Significantly, $100 \%$ of doctors surveyed would recommend the cards to future cohorts.

Lessons learnt The culture of reporting critical incidents has changed over recent years and most members of staff are aware of the importance of this in enabling change. We therefore noted that total number of critical incidents reported has dramatically increased over the last few years. This may have affected our data.

Message for others By encouraging access to guidelines, policies and procedures the cards help prevent clinical errors and promote patient safety. They alleviate stress and improve efficiency, enabling increased patient contact and clinical decision-making.

This cost-effective project could be expanded nationwide across departments and specialities, and our work to identify an objective measure of success will help others in making the case for change in their own departments.

\section{G525(P) CREATING A MEDICATION SAFETY CULTURE IN PICU}

${ }^{1}$ A Osman, ${ }^{2}$ D Panjwani, ${ }^{3} S$ Robinson. ${ }^{1}$ Paediatric Intensive Care Unit, University Hospital Southampton, Southampton, UK; ${ }^{2}$ Department of Paediatrics, University Hospital Coventry and Warwickshire, Coventry, UK; ${ }^{3}$ Paediatric Intensive Care Unit, University Hospitals of Leicester, Leicester, UK

\subsection{6/archdischild-2015-308599.476}

Context Two paediatric intensive care units within the same trust.

Problem Medication errors are a common, avoidable, occurrence, with significant associated morbidity and mortality. The aim of this project was to define the extent of the problem in our units and institute measures to reduce it.

Assessment of problem and analysis of its causes An anonymous audit was performed, which found numerous medication errors, with significant underreporting of errors occurring.

It was felt that the busy nature of the units contributed to the error rate, as staff were often unable to prescribe or administer medicines without interruption, leading to mistakes. There also seemed to be a general culture discouraging incident reporting, as staff felt that they were a tool for blame, with no benefit seen in completing them.

These findings highlighted the need for a culture change, from a 'blame culture' to one of 'fair accountability', with incident reporting seen as a tool for change, and staff given feedback on its positive outcomes. Staff also needed to treat medicine safety as a priority, with time, space and resources dedicated to empowering staff to say no to interruptions during prescribing and administration.

Intervention A dedicated prescribing area was set up, equipped with drug monographs, a BNF, a calculator and headphones to block out extraneous noise. A prescribing guideline was developed, instructing prescribers to use the dedicated area to write prescriptions without interruption. The guideline also instructed nurses to wear special aprons while preparing and administering medicines, protecting them from interruption.

Strategy for change Junior doctor and nursing involvement was a necessity in implementing this change, as they did the majority of the prescribing and administering. "Safety Champions" at each site were tasked with disseminating information, promoting good prescribing and administration habits and leading on-going audits. 